www.jmscr.igmpublication.org

Impact Factor 5.84

Index Copernicus Value: 83.27

ISSN (e)-2347-176x ISSN (p) 2455-0450

crossref DOI: https://dx.doi.org/10.18535/jmscr/v5i1.113

\author{
Journal Of Medical Science And Clinical Research \\ IGM Publication \\ An official Publication of IGM Publication
}

\title{
Elevation of Troponin-I in Sepsis \& Septic Shock
}

\author{
Authors \\ Dr Veena Santhoshi Avva, Dr. Vedavathi \\ KIMS Hospital
}

\begin{abstract}
The study of Elevation of troponin I in sepsis and septic shock on 40 patients was conducted in KIMS Hospital and research centre ICU, Bangalore during the period from November 2012 to May 2014. Cases were selected according to the sepsis criteria and enrolled for study. Objective: To determine the association of elevated cTnI levels in patients with sepsis and septic shock.

Methodology \& Results: Data were analyzed between sepsis and septic shock groups and between troponin I positive and negative groups. The average age groups in sepsis were 47.7 and in septic shock were 53.8. Males were mostly affected in both sepsis and septic shock groups (67\%) than females (32.5\%). ECG was normal in majority of patients and sinus tachycardia seen in majority of patients with septic shock. ECHO showed normal in all patients expect in two patients in septic shock group who had global hypokinesia. Majority of Patients were diagnosed with Pneumonia (45\%). Troponin I showed positive in 29(72.5\%) patients and normal in 11(27.5\%) patients which was statistically significant. Troponin I showed positive, more in septic shock group 19(95\%) as compared to sepsis group 10(50\%). In majority of cTnI positive patients $C K(18(62.0 \%)$ and CK-MB 17(58.6\%) was elevated which is not significant. Troponin I positive patients requiring ventillatory support $(82.7 \%)$, dialysis $(24.1 \%)$, ionotropic support (65.5\%) were more than patients in negative group requiring ventillatory support $(45 \%)$, dialysis $2(18.1 \%)$ and ionotropic support(9\%). In troponin I positive group from all cultures gram positive isolated was 11(63.6\%) and gram negative was $28(96.5 \%)$. In troponin I negative group from all culture gram positive isolated were $7(37.9 \%)$ and gram negative were 5(45.4\%). Isolation of gram positive and gram negative organisms in troponin I positive group was 39(134.4\%) compared to negative group which is 12(109.09\%). Isolation of fungi and virus (H1N1) in troponin I positive group showed 6(20.6\%) compared to negative group which is 1(90.9\%) showing statistically significant.

Conclusion: Elevation of troponin I in sepsis and septic shock indicates inflammatory and toxic damage to heart apart from ischemic damage caused by myocardial Infarction.
\end{abstract}

\section{Introduction}

Troponin I is a cardiac biomarker, it is elevated in Acute MI. It gives risk stratification and bad prognosis. It is also elevated in critically ill patients like sepsis and septic shock. It is also seen in pulmonary embolism, exacerbation of COPD, snake bite, Rhabdomyolysis.
In this study we have studied association of Trop I in sepsis and septic shock. It is observed in 31$80 \%$ of patients with SIRS, sepsis, septic shock. Its association in MI indicates irreversible ischemic damage. In sepsis the elevation is due to Inflammatory and reversible damage to the heart. The presence of cardiovascular dysfunction in 
sepsis is associated with increased mortality rate of $70 \%-90 \%$ compared with $20 \%$ in septic patients without cardiovascular impairment. Thus myocardial dysfunction in sepsis has been the focus of intense research activity. Myocardial injury is common in patients without acute coronary syndrome.

\section{Objective}

$>$ To determine the association of elevated cTnI levels in patients with sepsis and septic shock.

$>$ To detect Myocardial damage in patients without Acute Coronary Syndrome.

To determine cTnI values as an indicator for toxic and inflammatory damage to the heart apart from ischemic damage caused by MI.

\section{Methodology}

A total of 40 Patients aged >18years with a diagnosis of sepsis and septic shock admitted in KIMS Hospital and Research Centre from ICU were enrolled in the study. The study was conducted for a period of 18 months from November 2012 to May 2014, 40 Patients with prior history of heart disease and with ECG changes were excluded. Informed consent was obtained from patients relatives. Detailed history was taken in all the patients with respect to presenting complaints, Predisposing factors and accompanying illness. A thorough Clinical examination was carried out.

Investigations like CBC, Platelet Count, Serum electrolytes , RBS, ABG, RFT, LFT, PT, APTT, INR, Urine routine, ECG, ECHO, Cardiac Enzymes, Troponin I were done for all the patients on the day of admission. Troponin I were done by ELISA method. Chest X-ray, USGAbdomen and Pelvis, Blood Culture, Urine Culture, Swab Culture and Sputum Culture were analyzed for all the patients.

\section{Exclusion Criteria}

Patients with Acute Coronary Syndrome, Clinical Evidence of Congestive Cradiac failure, cardiac pulmonary resuscitation/defibrillation before admission. History of Previous Cardiac disease and patients with ECG Changes were excluded.

\section{Statistical Methods}

Categorical data is presented as numbers (percents) and continuous data as mean and median. Pearson Chi-test was used to compare categorical and continuous variables.

Chi square test

$x^{2}=\frac{\sum(O i-E i) 2}{E i}$ where $\mathrm{Oi}$ is observed frequently and $\mathrm{Ei}$ is expected frequency

$P$ value of $<0.01$ : very strong presumption against null hypothesis.

$0.01<\mathrm{p}<0.05$ :strong presumption against null hypothesis.

$0.05<\mathrm{p}<0.1$ :low presumption against null hypothesis.

p>0.1: no presumption against null hypothesis.

\section{Results}

Out of 40 individuals, 20 patients were with sepsis and 20 patients with septic shock were included in the study. In sepsis group, 15 male (75\%), 5 female $(5 \%)$ were seen which was statistically not significant. In septic shock group 12 males (60\%), 8 females $(40 \%)$ were seen which was statistically not significant. Mean age observed in sepsis group were 47.7 and septic shock was 53.8. In sepsis group ECG was normal in 11(55\%) Patients, LVH in $1(5 \%)$ patient and sinus Tachycardia in $8(40 \%)$ patients.

In septic Shock group ECG was normal in 2(10\%) patients, sinus tachycardia in $18(90 \%)$ patients. In sepsis group ECHO was normal in all 20 patients, (Mean EF-58\%, SD-0.047). In Septic shock group ECHO was normal in 18 patients, Global Hypokinesia was seen in 2 patients with EF of 48\%, 42\%. (Mean EF-57\%, SD-0.036). Total Mean EF was $58 \%$ with SD 0.0427. There was no significant difference of ECHO in sepsis and septic shock group. In Sepsis group mean PR was 


\section{JMSCR Vol||05||Issue||01||Page 15935-15943||January}

114.05 SD 7.990,mean RR 37.19 SD 4.106,mean SBP 121.70 SD 14.970,mean DBP 78.00 SD 8.944, Temparature 100.91 F, SD 0.701.

In septic shock group mean PR was 121.5 SD 9.942,RR 41.06 Sd 3.589,SBP 77.78 SD 20.452, DBP 54.38 SD 9.369, Temparature 100.53 SD 0.640. Out of 40 Patients 23 had Pre Existing Comorbidities, 17 had no co-morbidities.

In Sepsis, COPD+ Hypothyroidism was seen in one patient, DM in 6 Patients, HTN in 2 Patients, $\mathrm{DM}+\mathrm{HTn}$ in 2 Patients, HTN+OLDCVA in 1 patient,8 Patients without any co-morbidities. In septic Shock group, HTN was seen in 1 patient, COPD in 1 patient, DM in 3 Patients, DM+HTN in 6 Patients and 9 patients without any comorbidities.

In sepsis group 9 patients with pneumonia,4cellulitis, 1-urosepsis, 1-cholecysitis,mixed

infections like leptospirosis +urosepsis in 1 patient, Cellulitis+urosepsis in 1 patient, pneumonia+cellulitis in 1 patient, pneumonia+urosepsis in 2 patients were seen which was statistically not significant.

In septic shock group 9 patients with pneumonia, 3-celllulitis,2-urosepsis,1-dengue,mixed infections like cellulitis+urosepsis in 1 patient, pneumonia+urosepsis in 3 patients, dengue+urosepsis in 1 patient were seen which was statistically not significant.

\section{Troponin I category in sepsis and septic shock patients}

\begin{tabular}{|l|c|c|c|}
\hline \multirow{2}{*}{ DIAGNOSIS } & \multicolumn{2}{|c|}{ TROP I } & \multirow{2}{*}{ TOTAL } \\
\cline { 2 - 3 } & $\langle 0.017$ microgm/I & $>0.017$ microgm/I & \\
\cline { 2 - 3 } Sepsis & $10(50 \%)$ & $10(50 \%)$ & 20 \\
\hline \multirow{2}{*}{ Septic shock } & $1(5 \%)$ & $19(95 \%)$ & $50.0 \%$ \\
\cline { 2 - 3 } & 11 & 29 & $50.0 \%$ \\
\hline Total & $27.5 \%$ & $72.5 \%$ & 40 \\
\hline P Value & & & 0.001438 \\
\hline
\end{tabular}

Troponin I value of 0.017 mocrograms/I is taken as cutoff value from our lab. In Sepsis group, Troponin I was positive in $10(50 \%)$ patients and normal in 10(50\%) Patients. In septic shock group Troponin I was positive in $19(95 \%)$ patients and normal in 1(5\%) patient. Elevation of Troponin I was statistically significant with a $P$ value of 0.001438 showing a trend of increased levels in septic shock group.

No. of patients with elevated and normal value of troponin $I$ in sepsis and septic shock with a trend of increased levels in septic shock group.

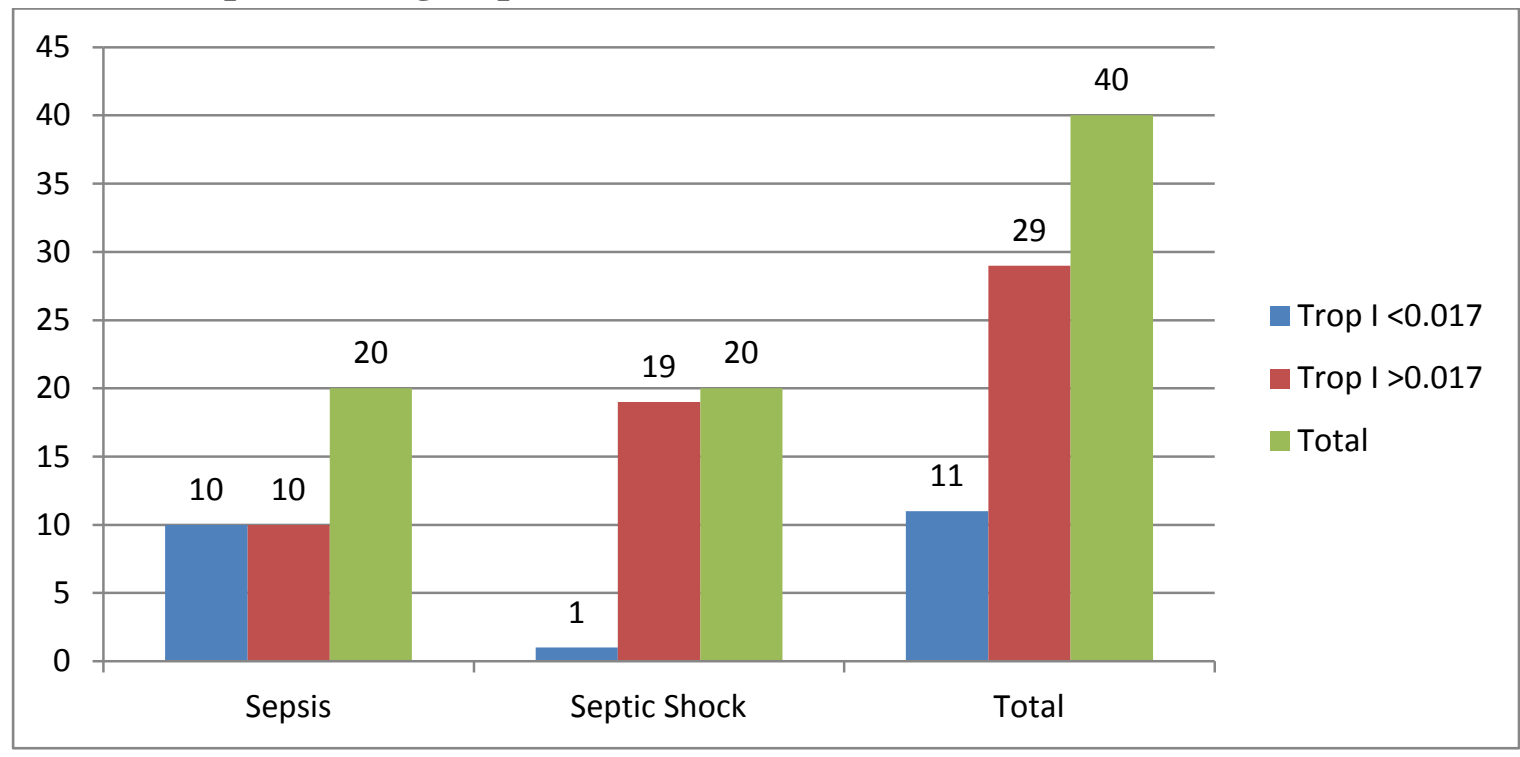




\section{JMSCR Vol||05||Issue||01||Page 15935-15943||January}

Troponin $I$ in comparison with Elevated Cardiac $C$ enzymes

\begin{tabular}{|l|c|c|c|c|c|c|}
\hline $\begin{array}{l}\text { Troponin I } \\
(<0.017)\end{array}$ & C K Positive & C K Negative & $\begin{array}{c}\text { Median of CK } \\
\text { in relation to } \\
\text { Trop I }\end{array}$ & $\begin{array}{c}\text { CK-MB } \\
\text { Positive }\end{array}$ & $\begin{array}{c}\text { CK-MB } \\
\text { Negative }\end{array}$ & $\begin{array}{c}\text { Median of CK- } \\
\text { MB in relation } \\
\text { to Trop I }\end{array}$ \\
\hline Positive(29) & $18(62.0 \%)$ & $11(37.9 \%)$ & 402,32 & $17(58.6 \%)$ & $12(41.3 \%)$ & $32,3.5$ \\
\hline Negative(11) & $5(45.4 \%)$ & $6(54.5 \%)$ & 172,25 & $1(9.09 \%)$ & $10(90.9 \%)$ & 5 \\
\hline Total(40) & $23(57.5 \%)$ & $17(42.5 \%)$ & & $18(45 \%)$ & $22(55 \%)$ & \\
\hline P Value & & 0.342558 & & & 0.004931 & \\
\hline
\end{tabular}

In troponin I positive group CK was elevated in $18(62.0 \%)$ patients, normal in $11(37.9 \%)$ patients. In troponin I negative group CK was elevated in $5(45.4 \%)$ patients, normal in $6(54.5 \%)$ patients. Out of 40 patients CK was elevated in $23(57.5 \%)$ patients, negative in 17(42.5\%) patients. Elevation of CK in relation to troponin I was statistically not significant with a $\mathrm{P}$ Value of 0.342558 . Median Total CK elevation in troponin I Positive patients was 402 as compared to 172 in troponin I negative patients.
In troponin I positive group CK-MB was elevated in $17(58.6 \%)$ and normal in $12(41.3 \%)$ patients. In troponin I negative group CK-MB was elevated in $1(9.09 \%)$ and normal in 10(90.9\%) patients. Out of 40 Patients CK-MB was elevated in 18(45\%) and normal in $22(55 \%)$ patients. Elevation of CKMB in relation to troponin I was statistically significant with a $\mathrm{P}$ value of 0.004931 . Median total CK-MB elevation in troponin I positive patients was 32 as compared to 1 in troponin I negative patients.

Elevation of cardiac enzymes in relation to Troponin I showing increased elevation in troponin I positive group.

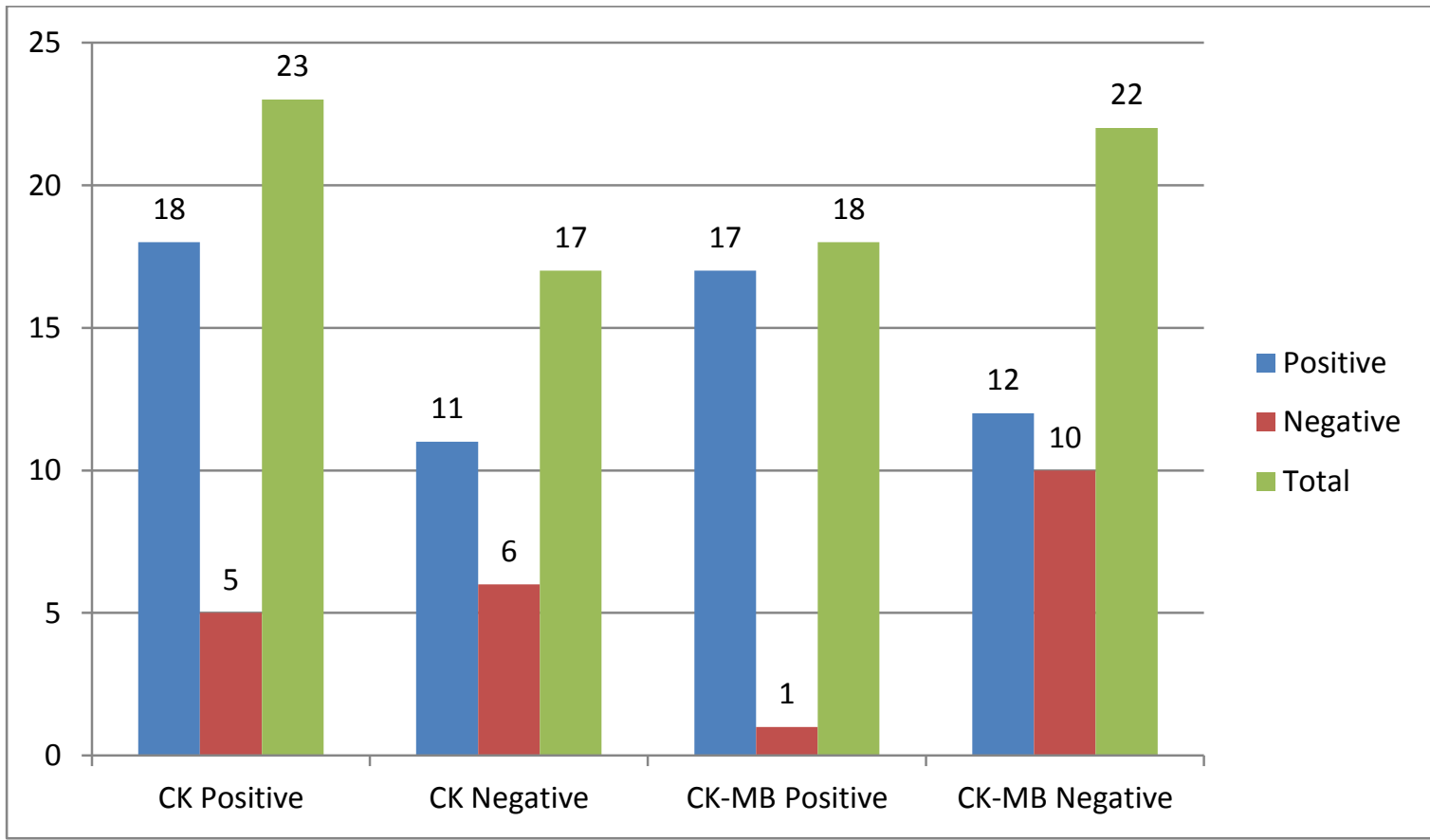

In troponin I positive group 24(82.7\%) patients required ventillatory support, $7(24.1 \%)$ patients required dialysis and $19(65.5 \%)$ required ionotropic support. In troponin I negative group $5(45 \%)$ patients required ventillatory support, $2(18.1 \%)$ patients required dialysis and $1(19 \%)$ required ionotropic support. Out of 40 patients $29(72.5 \%)$ patients required ventillatory support, $9(22.5 \%)$ patients required dialysis and $20(50 \%)$ required ionotropic support. Patients requiring ventillatory support in relation to troponin I elevation was statistically significant with a $P$ value of 0.018309 . 


\section{JMSCR Vol||05||Issue||01||Page 15935-15943||January}

Patients requiring ionotropic support in relation to troponin I elevation was statistically significant with a $\mathrm{P}$ value of 0.001438 , Whereas patients requiring dialysis in relation to troponin $\mathrm{I}$ elevation was statistically not significant with a $\mathrm{P}$ value of 0.687098 .

Patients requiring ventillatory support, dialysis and ionotropic support in relation to troponin I

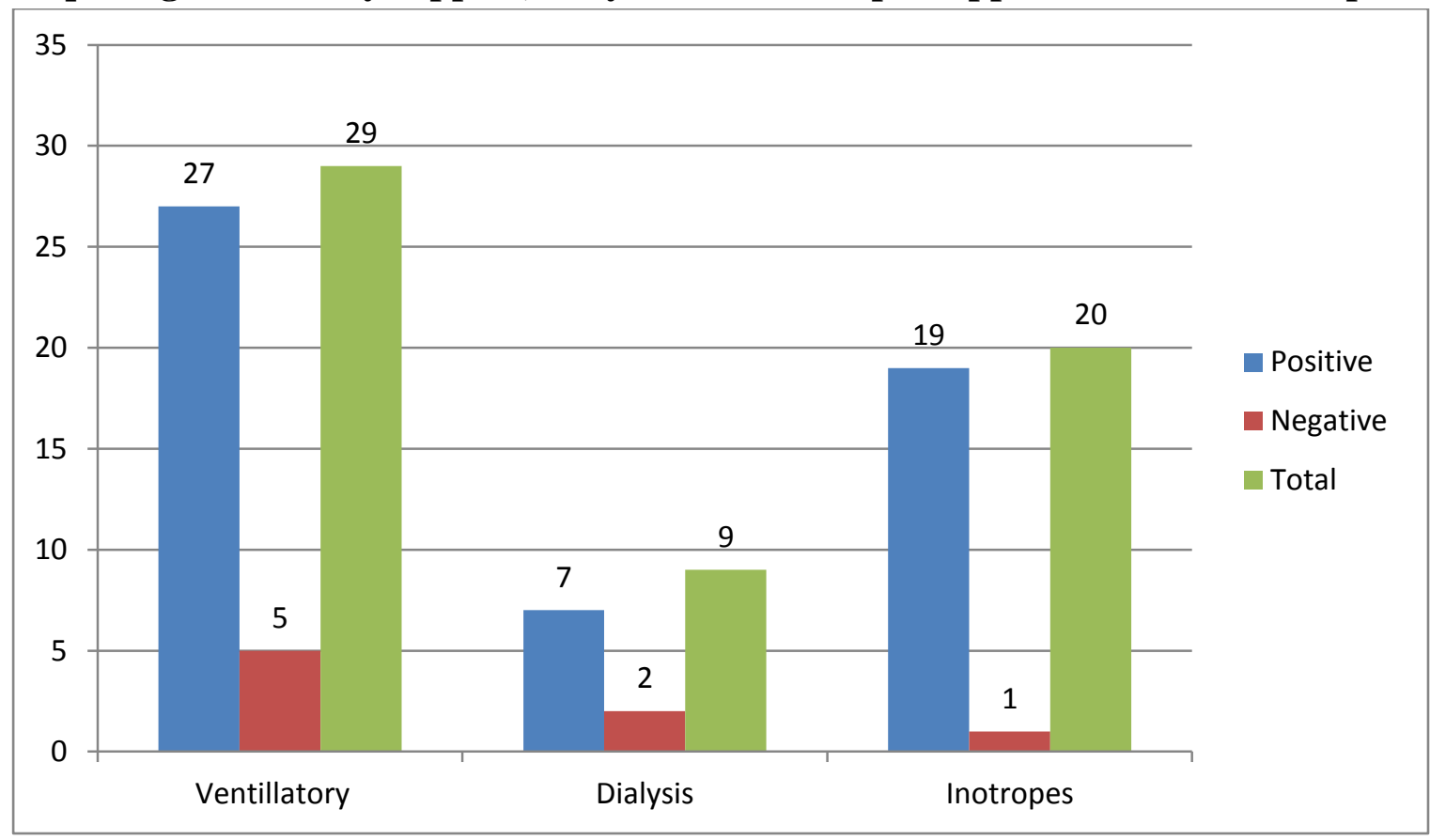

Inotropes I in relation with type of Pathogen Isolated in Sputum Culture

\begin{tabular}{|l|c|c|c|c|c|}
\hline TROPONIN I & $\begin{array}{c}\text { GRAM } \\
\text { POSITIVE }\end{array}$ & $\begin{array}{c}\text { GRAM } \\
\text { NEGATIVE }\end{array}$ & VIRAL (H1N1) & FUNGAL & TOTAL \\
\hline POSITIVE & $4(13.7 \%)$ & $15(51.7 \%)$ & $1(3.4 \%)$ & $1(3.4 \%)$ & $21(72.4 \%)$ \\
\hline NEGATIVE & $1(9.09 \%)$ & $2(18.1 \%)$ & 0 & $1(9.09 \%)$ & $4(36.3 \%)$ \\
\hline TOTAL & $5(12.5 \%)$ & $17(42.5 \%)$ & $1(2.5 \%)$ & $2(5 \%)$ & $25(62.5 \%)$ \\
\hline P VALUE & & & & & 1.2777 \\
\hline
\end{tabular}

In Troponin I positive group gram positive isolated was $4(13.7 \%)$, gram negative $15(51.7 \%)$, viral (H1N1) $1(3.4 \%)$ and fungi 1(3.4\%). In troponin I negative group gram positive was isolated in $1(9.09 \%)$, gram negative in $2(18.1 \%)$, fungal in $1(9.09 \%)$ patients. In troponin I Positive group total no. of pathogens isolated were $21(72.4 \%)$ as compared to $4(36.3 \%)$ in troponin I negative group which was not statistically significant with a $\mathrm{P}$ value of 1.2777.

\section{Troponin I in relation with Type of Pathogen} isolated in urine culture

In troponin I positive group gram positive was not isolated, gram negative isolated was 6(20.6\%), and fungi was $2(6.89 \%)$ patients. In troponin I negative group gram positive isolated was 


\section{JMSCR Vol||05||Issue||01||Page 15935-15943||January}

Type of pathogens isolated from urine culture in relaation to troponin I positive and negative groups where the pathogens isolated in positive group were more than the negative group.

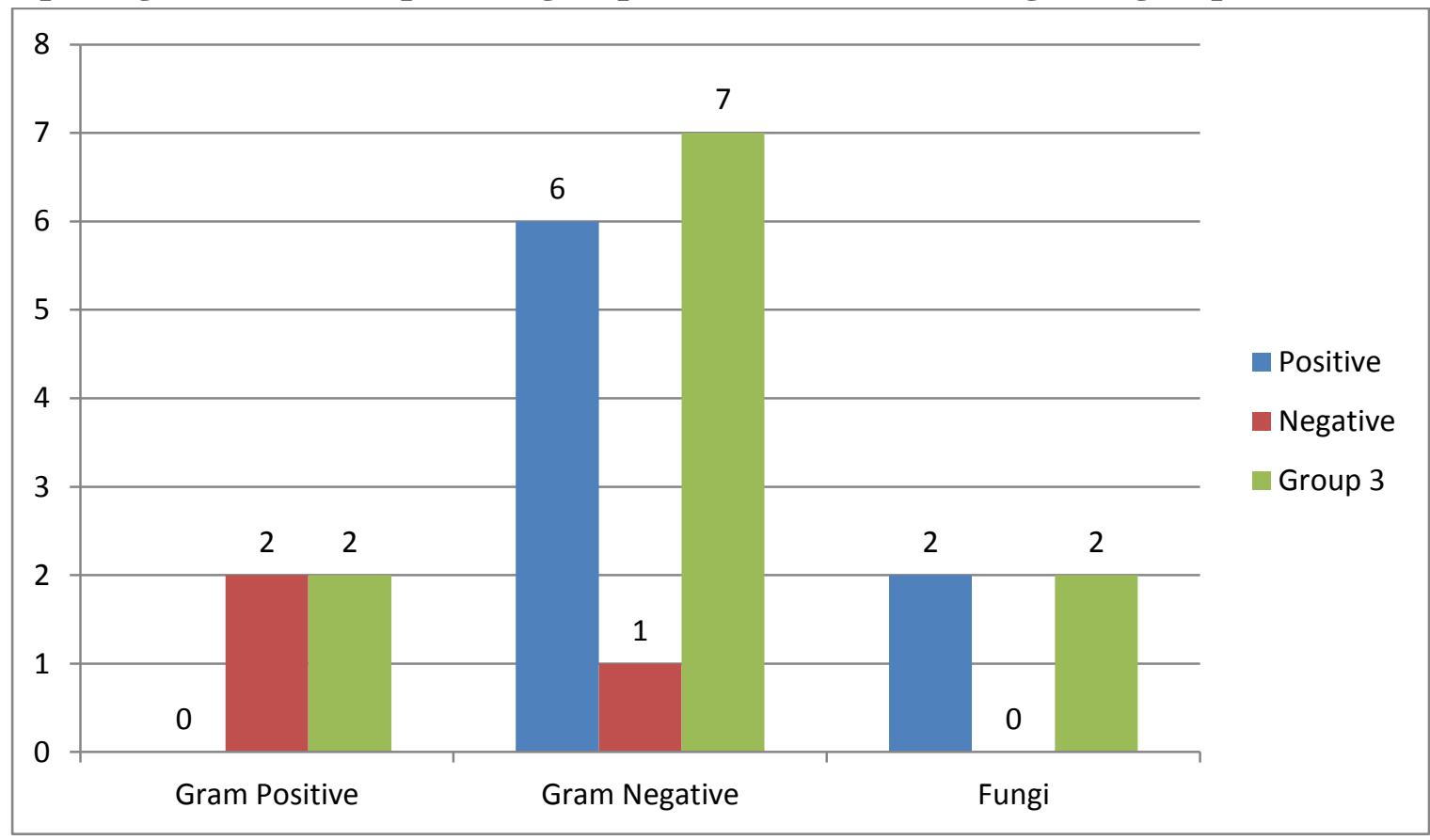

Troponin $I$ in relation with type of pathogens isolated in Blood Culture

\begin{tabular}{|l|c|c|c|c|}
\hline TROP I & GRAM POSITIVE & GRAM NEGATIVE & FUNGAL & TOTAL \\
\hline POSITIVE & $4(13.7 \%)$ & $4(13.7 \%)$ & $2(6.89 \%)$ & $10(34.4 \%)$ \\
\hline NEGATIVE & $1(9.09 \%)$ & $1(9.09 \%)$ & 0 & $2(18.1 \%)$ \\
\hline TOTAL & $5(12.5 \%)$ & $5(12.5 \%)$ & $2(5 \%)$ & $12(30 \%)$ \\
\hline P VALUE & & & & 0.000395 \\
\hline
\end{tabular}

In troponin I positive group gram positive isolated was $4(13.7 \%)$, gram negative $4(13.7 \%)$, and fungi $2(6.89 \%)$. In troponin I negative group gram positive isolated was $1(9.09 \%)$, gram negative $1(9.09 \%)$, fungi was not isolated. In troponin I positive group total no. of pathogens isolated were $10(34.4 \%)$ as compared to $2(18.1 \%)$ in troponin I negative group which was statistically significant with a $P$ value of 0.000395 .

In troponin I positive group gram positive isolated weres $3(10.3 \%)$ and gram negative was $3(10.3 \%)$. In troponin I negative group gram positive was isolated in $3(27.2 \%)$ and gram negative was $1(9.09 \%)$. In troponin I positive group total no. of pathogens isolated were $6(20.6 \%)$ as compared to $4(36.3 \%)$ in troponin I negative group which was statistically not significant with a $\mathrm{P}$ value of 0.429195 .

\section{Type of Pathogen isolated in All Culture}

In troponin I positive group from all culture gram positive isolated was 11(63.6\%) and gram negative was $28(96.5 \%)$. In troponin I negative group from all culture gram positive isolated was $7(37.9 \%)$ and gram negative was $5(45.4 \%)$. Isolation of gram positive and gram negative organisms in troponin I positive group was $39(134.4 \%)$ compared to negative group which was $12(109.09 \%)$ which was statistically significant with a $\mathrm{P}$ Value of 0.05

In troponin I positive group from all cultures fungi isolated was $5(17.2 \%)$ and Virus(H1N1) was $1(3.4 \%)$. In troponin I negative group from all culture fungi isolated was $1(9.09 \%)$ and virus (H1N1) was not isolated. Isolation of fungi and virus $(\mathrm{H} 1 \mathrm{~N} 1)$ in troponin I positive group was $6(20.6 \%)$ compared to negative group which was $1(9.09 \%)$ which was statistically significant with a $\mathrm{P}$ Value of 0.021161. 
Type of Pathogen isolated in all culture where more number of pathogens are isolated in Troponin I Positive group compared to Negative group.

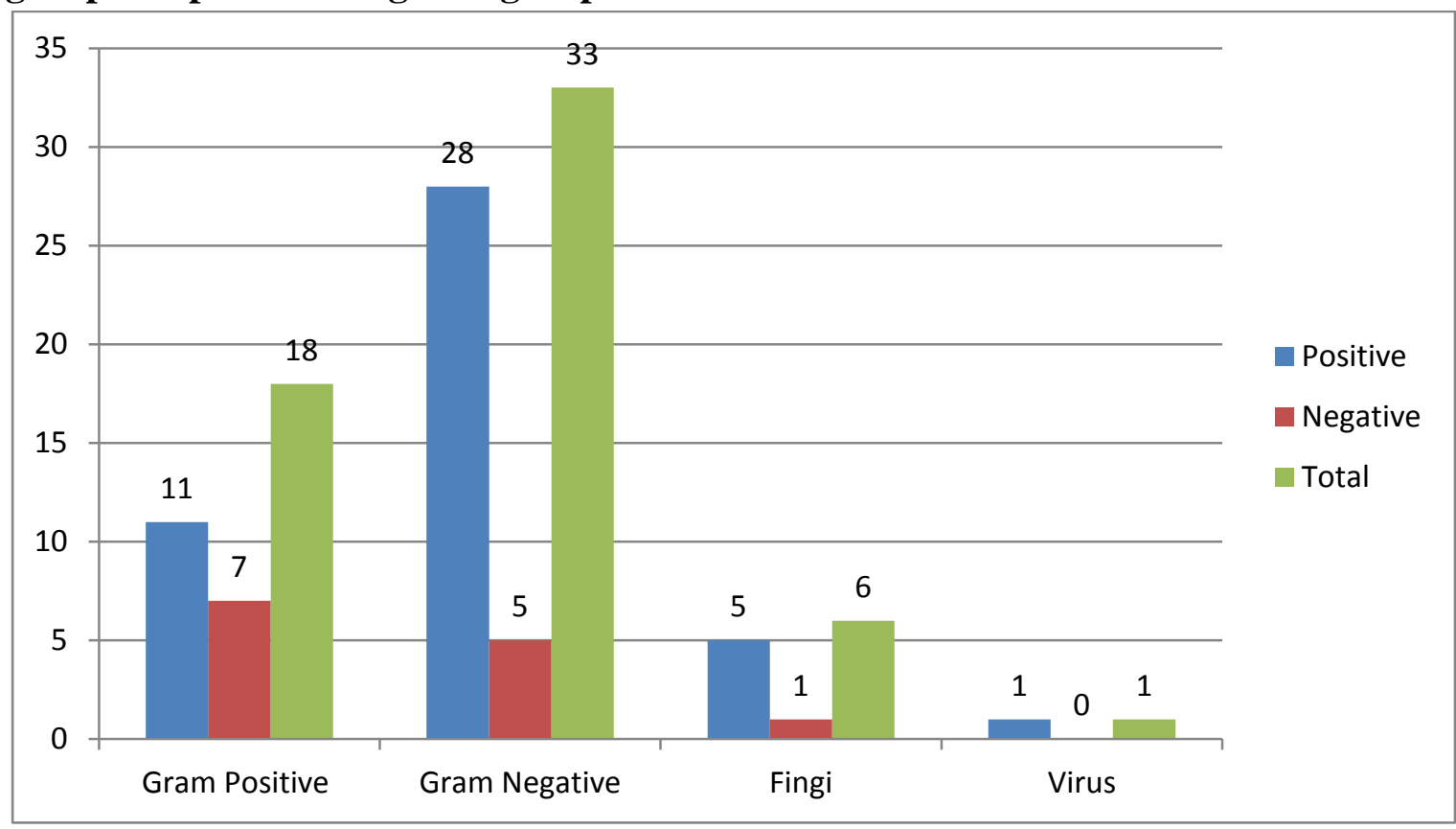

\section{Discussion}

In AMMAN etal Study. Troponin I in sepsis and septic shock were positive in $85 \%$ patients with a $\mathrm{p}$ value 0.0001 which showed majority of patients with sepsis and septic shock had elevated cTnI levels indicating inflammatory myocardial damage to heart in sepsis. ${ }^{8}$ In present study Troponin I is positive in $72.5 \%$ of patients with a p value of 0.001438 which is significant. ${ }^{8}$

In AMMAN etal study among cTnal positive patients gram positive pathogens isolated were $53 \%$ and gram negative pathogens isolated were $25 \%$ whereas in present study gram positive pathogens isolated were $63.6 \%$ and gram negative pathogens isolated were $96.5 \% .^{8}$

In AMMAN etal, median total CK elevation in troponin I positive patients was $411 \mathrm{U} / \mathrm{L}$, median total CK-MB elevation in troponin I positive patients was 33U/L. In present study median total $\mathrm{CK}$ elevation in troponin I positive patients was 402U/L, median total CK-MB elevation in troponin I positive patients was $32 \mathrm{U} / \mathrm{L}$.

In David R. Altmann st.al study pneumonia was seen in $14(37 \%)$ patients, Cholecystitis in 3(8\%) pts, Urospepsis in $1(3 \%)$ pts whereas in present study pneumonia was seen in $18(45 \%)$ patients, Cholecystitis in $1(5 \%)$ patients, Urosepsis in 3
(7.5\%) $\mathrm{pts}^{4}$. In David R Altmann et.al study gram positive organisms isolated was $15(40 \%)$, gram negative organisms isolated was $11(29 \%)$, fungi was $2(5 \%)^{(4)}$ whereas in present study gram ppositive organisms isolated was $18(45 \%)$ gram negative was $33(82.5 \%)$ and fungi was $6(15 \%)$. In David R.Altmann et.al ${ }^{9}$ study cTnI positive patient requiring ventilator support was 14(64\%) and troponin I negative group was $8(50 \%)$ with a $\mathrm{P}$ value 0.51 . In David R.Altmann et.al study cTnI positive patients requiring dialysis was $10(45 \%)$ and negative group 6(38\%) with a $\mathrm{P}$ value $0.74 \mathrm{In}$ David R.Altmann et.al study the need for mechanical ventilation and dialysis did not differ in troponin I positive and negative groups. In present study cTnI positive patient requiring ventilator support was $24(82.7 \%)$ and negative group requiring was $5(45 \%)$ which was statistically significant with a $\mathrm{P}$ value 0.018309 . In the present study cTnI positive patients requiring dialysis dialysis were $7(24.1 \%)$ and negative group was $2(18.1 \%)$ which was statistically not significant with a $\mathrm{P}$ value of 0.687098 .

In Mehta NJ et.al study CTnI positive patients requiring ionotropic support were $94 \%$ and in cTnI normal patients were $53 \%$ with a $\mathrm{P}$ Value of 0.018 indicating increased morbidity and 
mortality. In Present Study cTnI positive patients requiring ionotropic support was $65.5 \%$ and in cTnI normal patients was $9 \%$ with a $\mathrm{P}$ Value 0.001438 which is significant.

In this study, we have studied association of troponin I levels in sepsis and septic shock. Troponin I, a cardiac biomaker which is elevated in Acute Myocardial Infarction is also associated to be elevated in critically ill patients like sepsis and septic shock which is observed in $72.5 \%$ of patients in our study which was statistically significant (P Value 0.0014378).95\% of patients with septic shock had elevated troponin I levels which indicates ongoing severe myocardial depression caused by various mechanisms of sepsis, which indicates increased morbidity and close monitoring of these patients is required. $82.7 \%$ patients with elevated troponin I had ventillatory support (P Value 0.018309) which was statistically significant indicating increased morbidity. $65.5 \%$ patients had ionotropic support which was statistically significant (P Value 0.001438), which indicates increased morbidity.

In troponin I Positive group CK was elevated in $18(62.0 \%)$ patients of patients. Elevation of CK in relation to Troponin I was statistically not significant with a $\mathrm{P}$ value of 0.342558 . In troponin I Positive group CK-MB was elevated in $17(58.6 \%)$ patients. Elevation of CK-MB in relation to troponin I was statistically significant with a $P$ value of 0.004931 .

This shows that cardiac enzyme elevation is not independent of troponin I elevation but it is not specific and significant as it can be elevated in other conditions like muscle injury, trauma, renal failure etc.and also cardiac specificity compared to troponin I is less.

96.5\% of patients with gram negative and gram positive bacteria were isolated in troponin I positive which was statistically significant $(\mathrm{P}$ Value 0.05) which indicates that there could be endotoxin associated myocardial depression as it is one of the significant exogenous myocardial depressant substance released by gram negative bacteria proposed to cause myocardial depression, lower svr, lower mean EF by activating inflammatory mediators and inflammatory cascade and similar inflammatory damage by other mechanisms by gram positive bacteria.

Isolation of fungi and virus (H1N1) also contribute to elevation of troponin I by activating inflammatory mediators was also statistically significant ( $\mathrm{P}$ Value 0.021161). It indicates that cTnI is not only associated in myocardial infarction which is an indicator of irreversible ischemic damage, it can be elevated in conditions that cause inflammatory, toxic reversible damage to heart. It also indicates increased morbidity. ECG, ECHO may be normal in early stage of sepsis and septic shock when the underlying myocardial damage is undergoing.

Troponin I had greater sensitivity for minor degree of myocardial injury and also its isoform encoded by specific genes unique to myocardium makes it, a more reliable indicator to detect myocardial damage seen in majority of patients in sepsis and septic shock. Diagnostic test is also quick, convenient and cost effective.

Abnormalities of Cardiac function are frequent in patients with sepsis. Approximately 50\% of patients with severe sepsis and septic shock may develop impairment of ventricular performance. Whereas evaluation of myocardial performance during septic shock is of critical importance to select the best therapeutic options, several factors complicate the diagnosis of sepsis induced myocardial dysfunction. Recently, Plasma cardiac troponin has been proposed has a biomaker that accurately detects myocardial dysfunction and provides prognosis information in septic patients.

\section{Conclusion}

Elevation of troponin I in sepsis and septic shock indicates inflammatory and toxic damage to heart apart from ischemic damage caused by MI. Thus, it can be used as a marker for silent myocardial damage seen in sepsis and septic shock which may not be picked up by ECG and ECHO in early stages. 


\section{References}

1. Andrew $\mathrm{R}$ Chapman. Assessment and classification of patients with myocardial injury and infarction in clinical practice. Heart 2017;103:10-18

2. Alfredo Bardají. Troponin Elevation in Patients Without Acute Coronary Syndrome. Rev Esp Cardiol. 2015;68:469-76

3. Jaffe AS, Ravkilde J, Roberts R, et al. It's time for a change to a troponin standard. Circulation. 2000; 102: 1216-1220.

4. Myocardial infarction redefined: a Consensus document of The Joint European Society of Cardiology/American College of Cardiology Committee for the redefinition of myocardial infarction. J Am Coll Cardiol. 2000; 36: 959-969.

5. Hamm CW, Braunwald E. A classification of unstable angina revisited. Circulation. 2000; 102: 118-122

6. Bertrand ME, Simoons ML, Fox KAA, et al. Management of acute coronary syndromes: acute coronary syndromes without persistent ST segment elevation: recommendations of the Task Force of the European Society of Cardiology. Eur Heart J. 2000; 21: 1406-1432.

7. Braunwald E, Antman EM, Beasley JW, et al. ACC/AHA guidelines for the management of patients with unstable angina and non-ST-segment elevation myocardial infarction: executive summary and recommendations: a report of the American College of Cardiology/American Heart Association task force on practice guidelines (committee on the management of patients with unstable angina). Circulation. 2000; 102: 11931209

8. Amman P, Maggiorini M, Bertel O, et al. Troponin as a risk factor for mortality in critically ill patients with acute coronary syndrome. J Am Coll Cardiol. 2003; 41 (11):2004-2009.
9. Elevated Cardiac Troponin I in sepsis and septic shock: No Evidence for Thrombus Associated Myocardial Necrosis. David R Altmann, Korte W, Maeder MT, Fehr T, Haager P, et al. PLoS ONE. 5(2) February 32010. 